\title{
The Effect of Hippotherapy on Quality of Life in Women with Multiple Sclerosis
}

\section{Maryam Salehi*, Hasan Mohammadzadeh, Fahimeh Rezaei}

Department of Motor Behavior, Faculty of Sport Sciences, University of Urmia, Urmia, Iran

\section{Article Info:}

\section{ABSTRACT}

Introduction: The aim of this study was to examine the effect of hippotherapy on quality of life in women with multiple sclerosis. Materials and Methods: This study is a semiexperimental study with pre-test and post-test and a control group. Among patients with multiple sclerosis, 24 patients from a clinical center in Isfahan were recruited through convenient sampling and randomly and put in the experimental and control groups. In the pre-test step, participants filled out Multiple Sclerosis Quality of Life-54 Questionnaire. Then, the experimental group practiced hippotherapy training for 8 weeks ( 3 days in a week for 30 minutes). After completing the intervention, subjects in both groups participated in the post-test. Results: A significant difference between the mean scores of the experimental and control groups in physical and mental health dimensions of quality of life was observed. Conclusion: Our data indicate that hippotherapy training has a positive effect on the quality of life of women with multiple sclerosis.

*Corresponding Author: Maryam Salehi

E-mail:maryam_salehi288@yahoo.com 


\title{
اثر يك دوره اسب سوارى درمانى بر كيفيت زندكى زنان مبتلا به مالتيِّل اسكلروز
}

\author{
مريم صالحى"، حسن محمدزاده، فهيمه رضائى \\ كروه رفتار حركتى، دانشكده علوم ورزشى، دانشعاه اروميه، اروميه، ايران
}

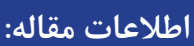

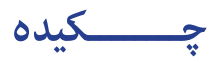

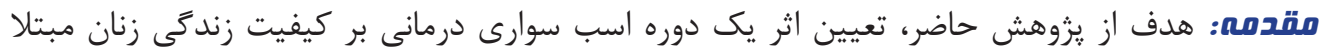

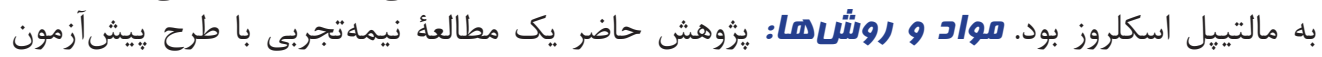

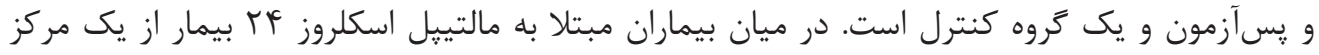

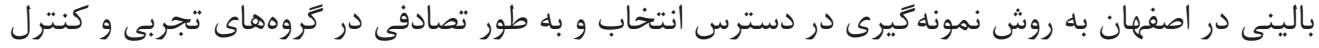

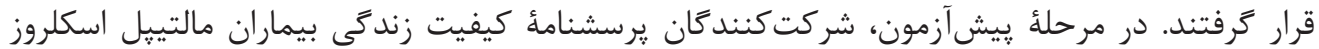

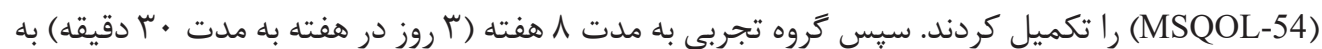

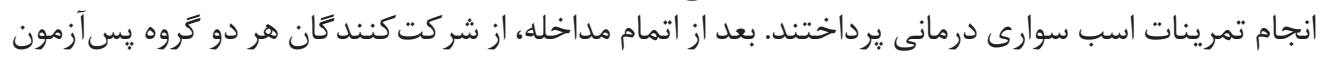

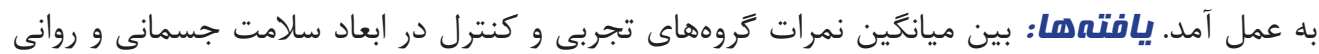

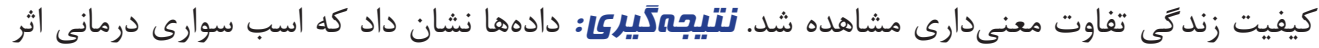
مثبتى بر كيفيت زندكى زنان مبتى مبتلا به مالتيِيل اسكلروز دارد.

كليد وازمها:

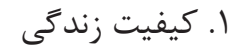

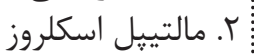

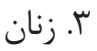

" نويسنده مسئول: مريم صالحى آدرس الكترونيكى: maryam_salehi288@yahoo.com 


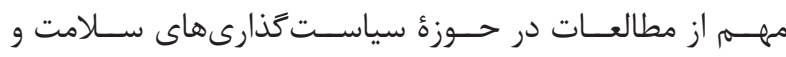

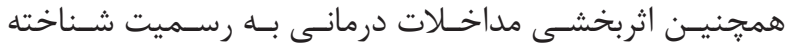

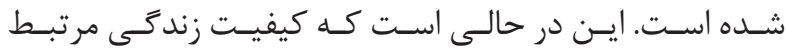

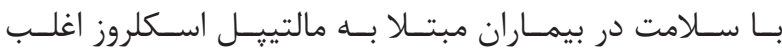

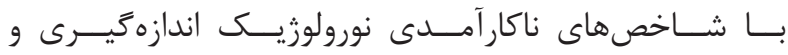

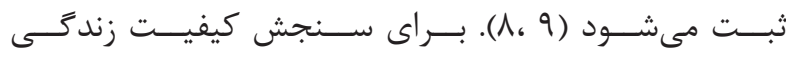

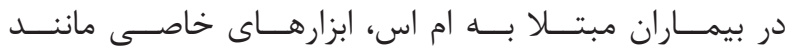

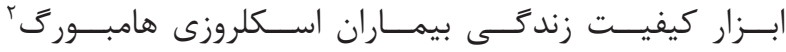

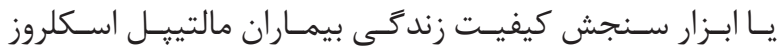

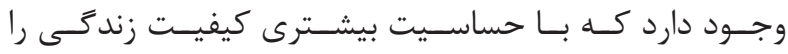

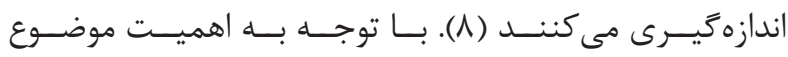

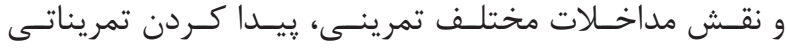

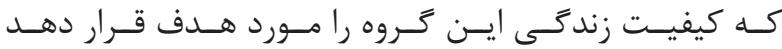

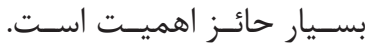

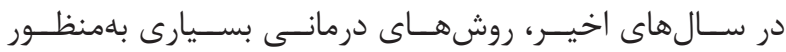

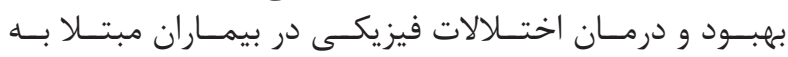

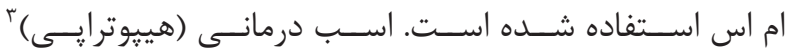

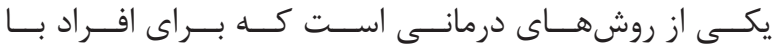

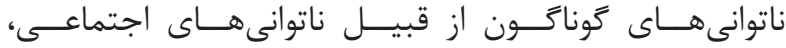

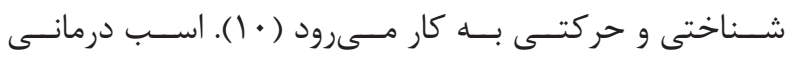

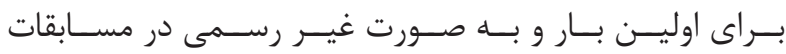

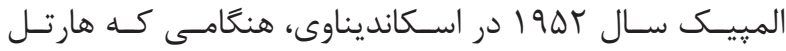

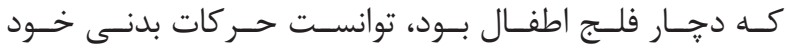

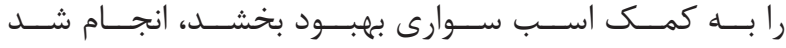

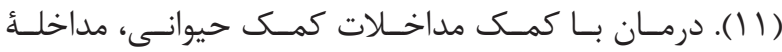

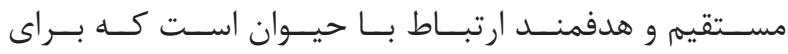

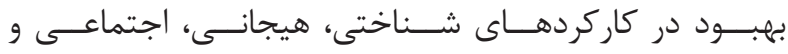

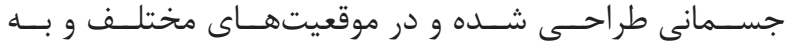

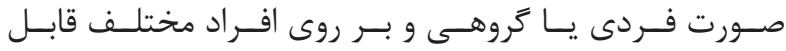

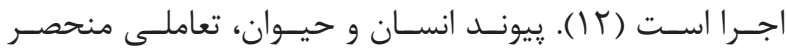

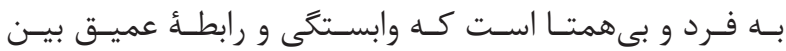

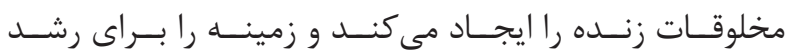

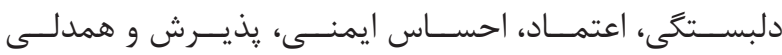

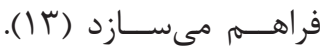

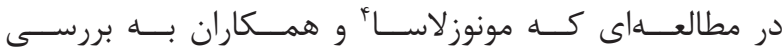

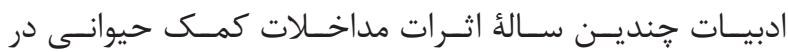

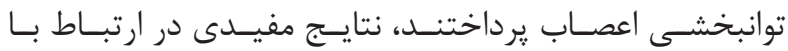

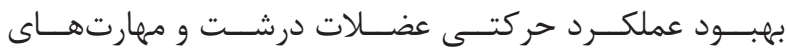

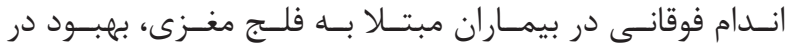

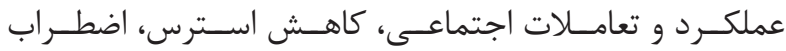

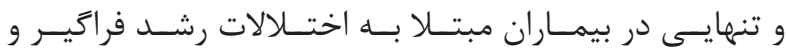

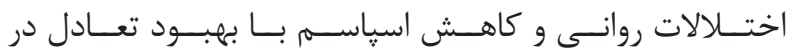

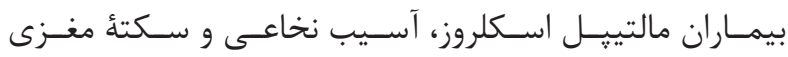

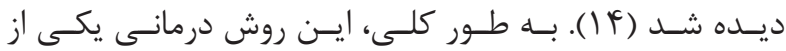

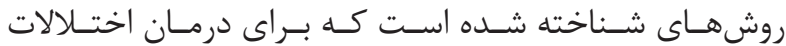

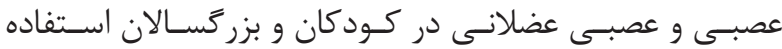

${ }^{1}$ Multiple sclerosis

${ }^{2}$ Hamburg
مقدمه

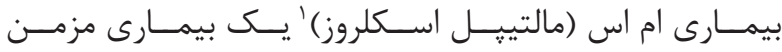

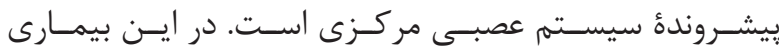

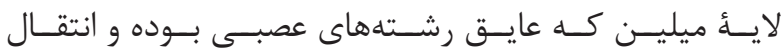

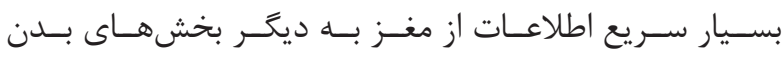

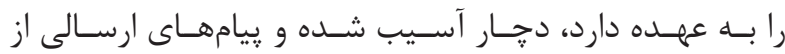

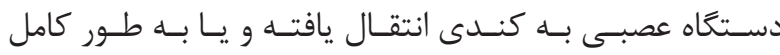

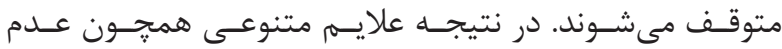

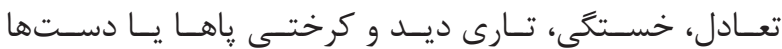

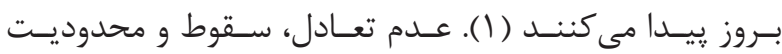

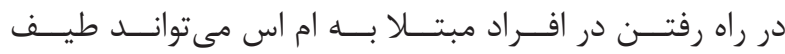

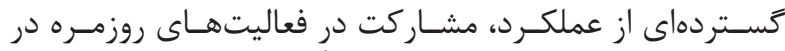

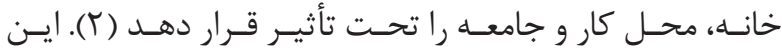

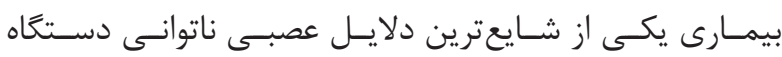

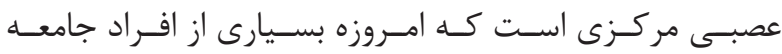

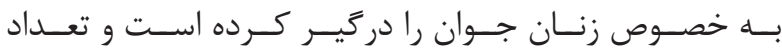

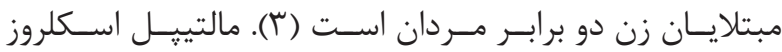

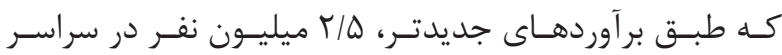

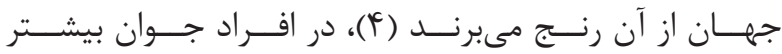

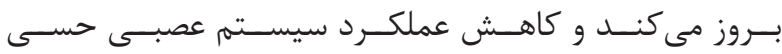

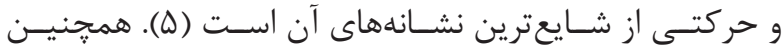

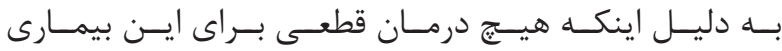

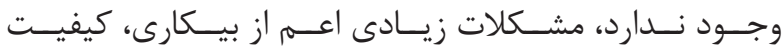

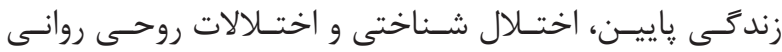

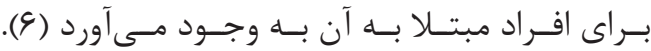

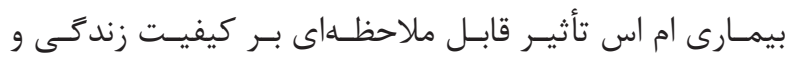

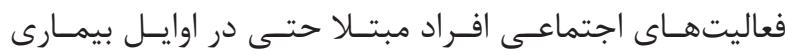

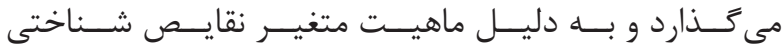

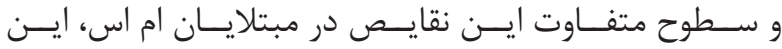

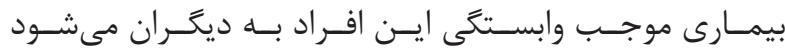

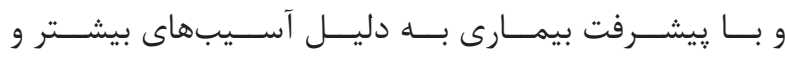

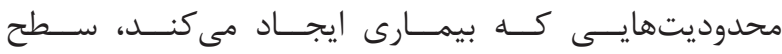

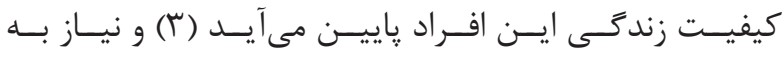

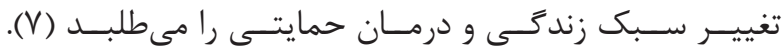

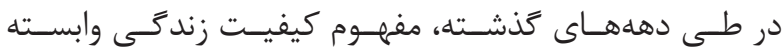

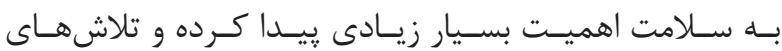

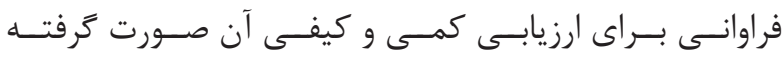

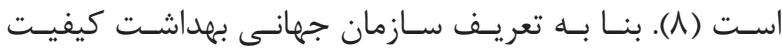

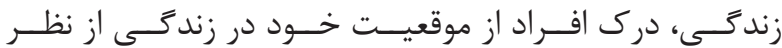

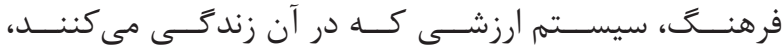

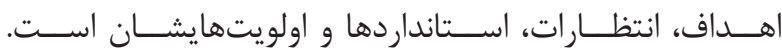

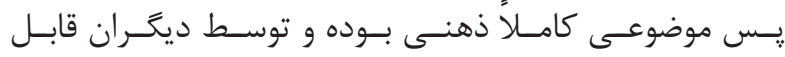

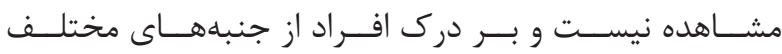

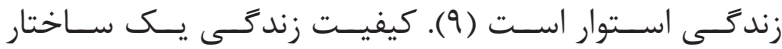

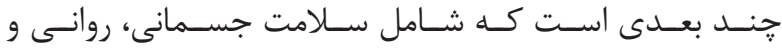

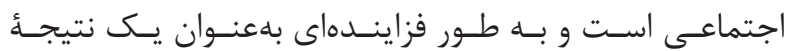

\footnotetext{
${ }^{3}$ Hippotherapy

${ }^{4}$ Munoz Lasa
} 


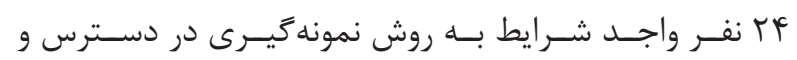

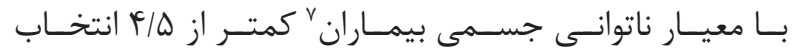

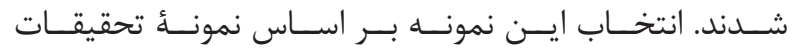

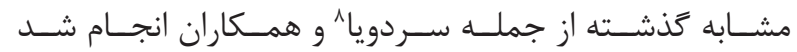

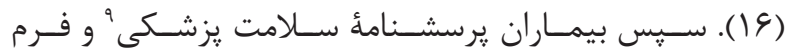

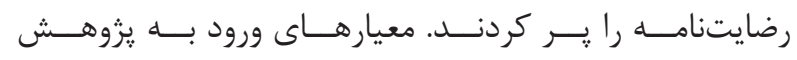

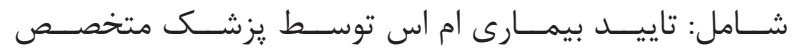

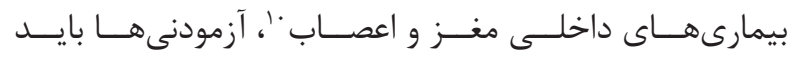

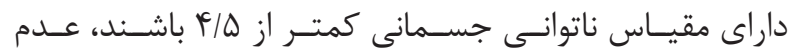

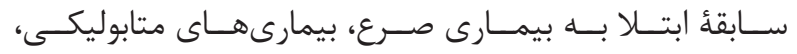

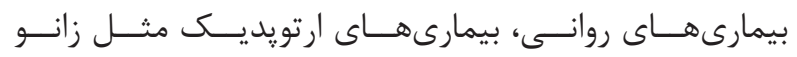

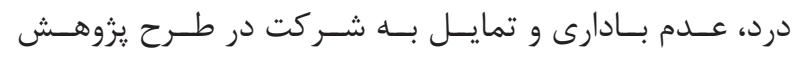

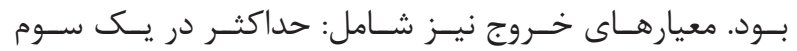

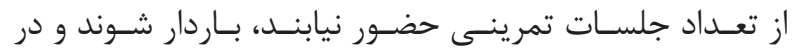

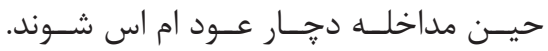

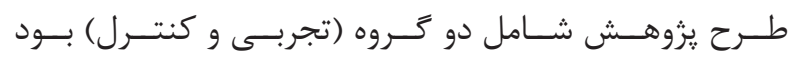

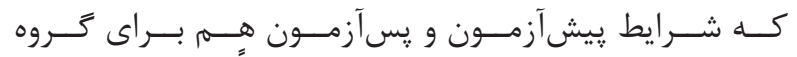

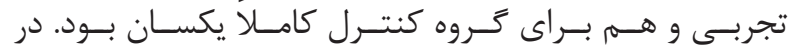

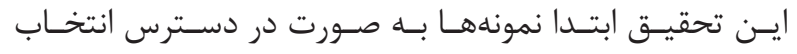

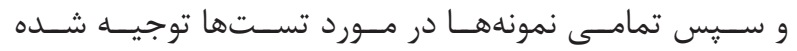

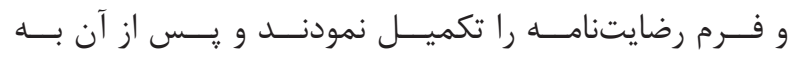

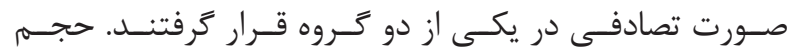

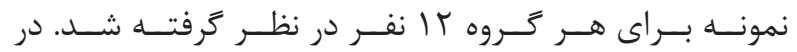

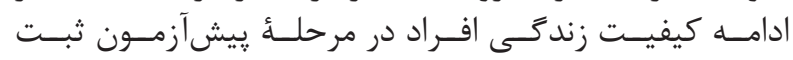

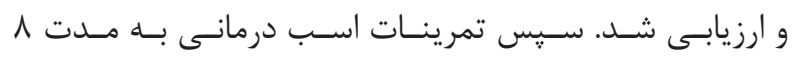

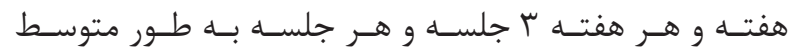

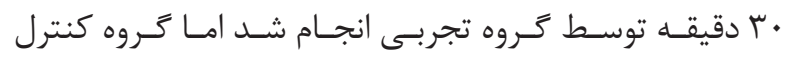

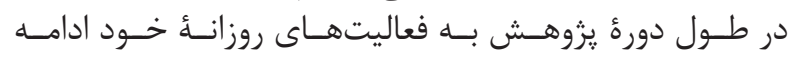

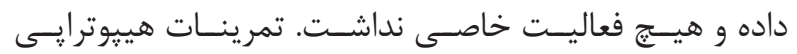

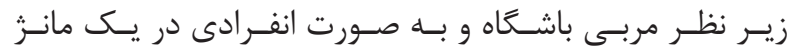

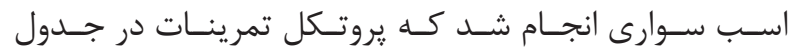

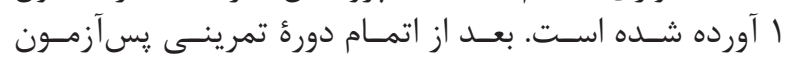

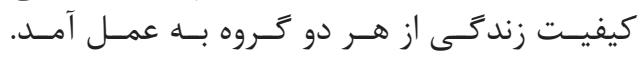

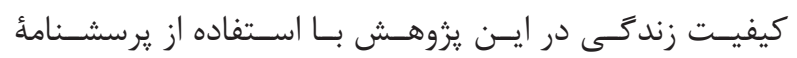

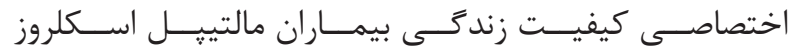
(MSQOL-54)

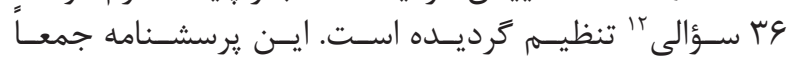

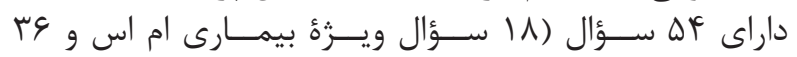

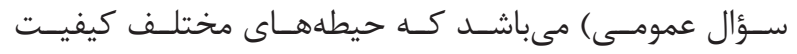

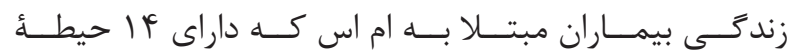

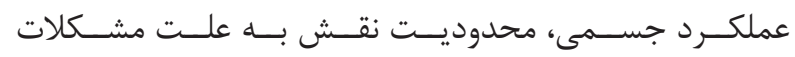

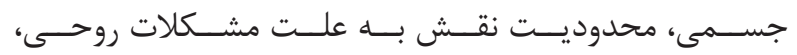

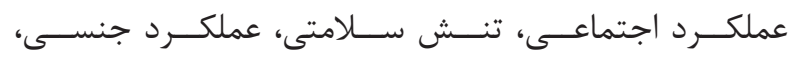

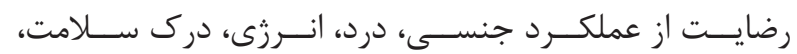

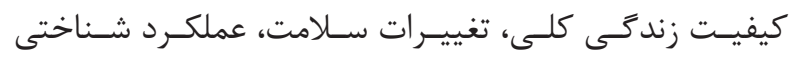

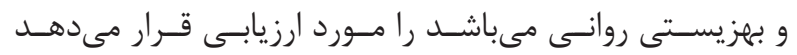

${ }^{5}$ Strock

${ }^{6}$ Wollenweber

${ }^{7}$ EDSS: expanded disability status scale

${ }^{8}$ Seredova

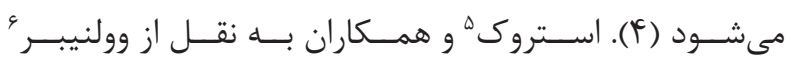

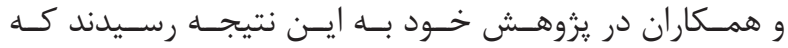

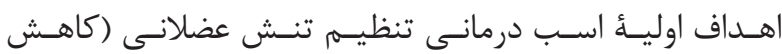

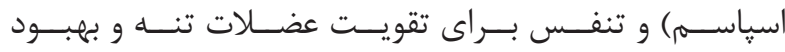

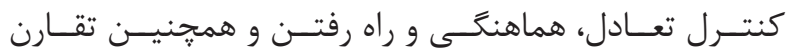

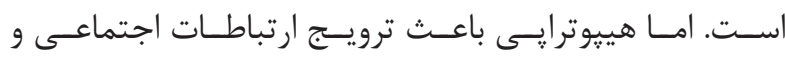

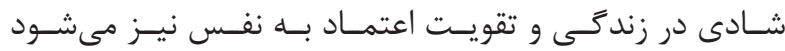

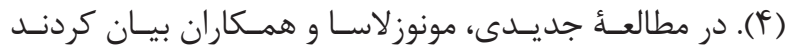

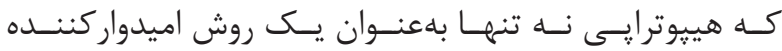

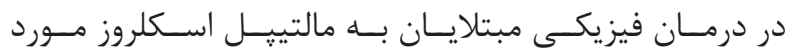

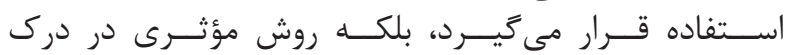

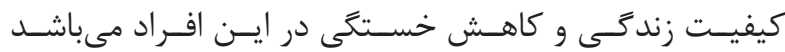

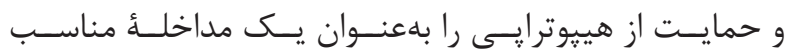

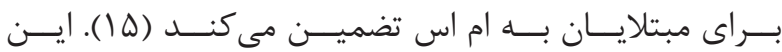

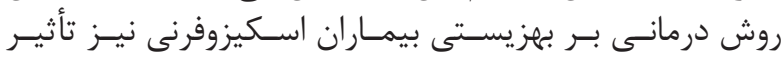

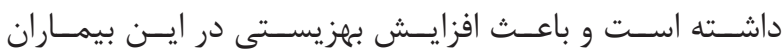

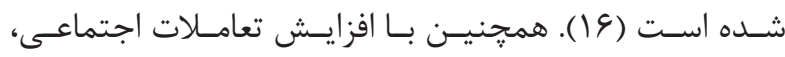

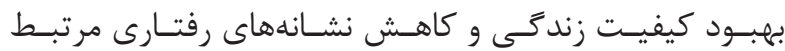

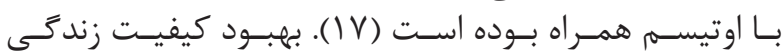

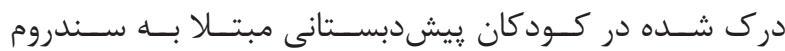

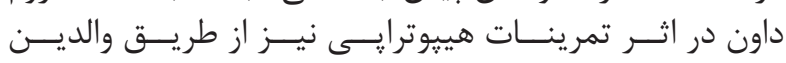

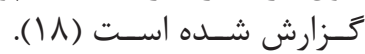

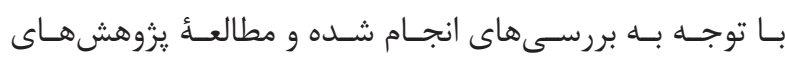

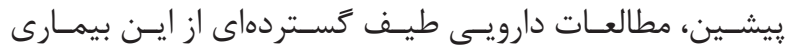

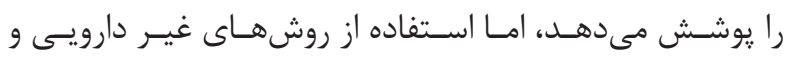

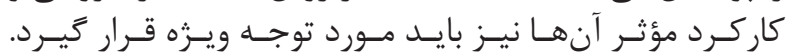

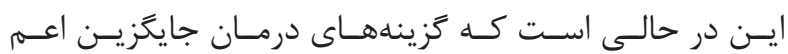

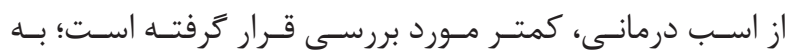

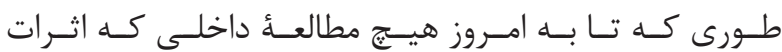

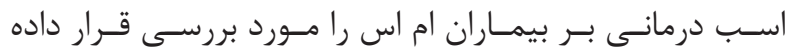

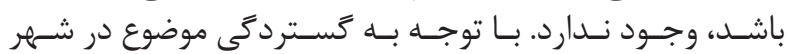

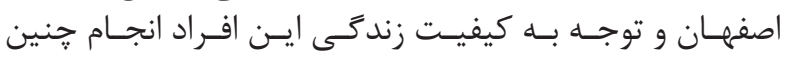

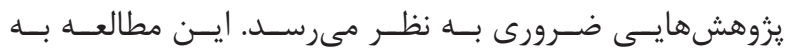

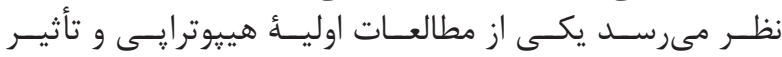

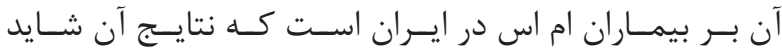

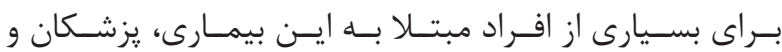

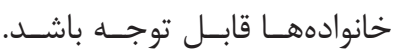

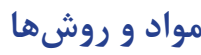

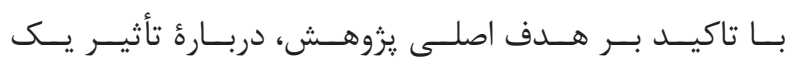

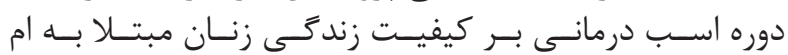

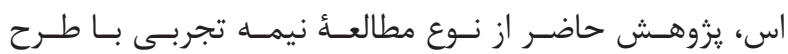

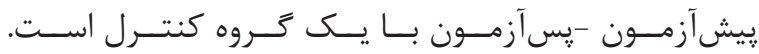

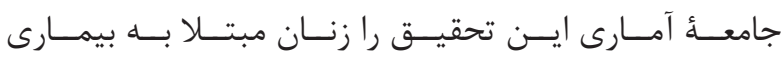

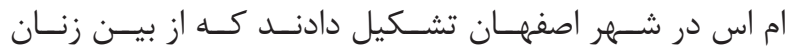

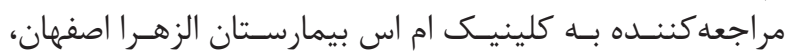

${ }^{9}$ PAR-Q

${ }^{10}$ Neurologist

${ }^{11}$ Multiple sclerosis quality of life-54

${ }^{12}$ SF-36 


\begin{tabular}{|c|c|}
\hline \multicolumn{2}{|c|}{ جدول ا- محتواى جلسات تمرينات با اسب. } \\
\hline محتواى جلسات & جلسه \\
\hline 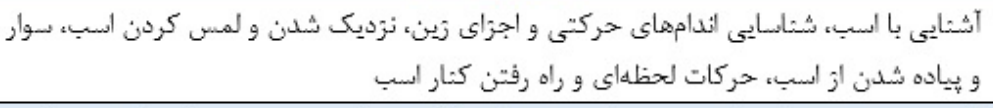 & هفتة اول \\
\hline 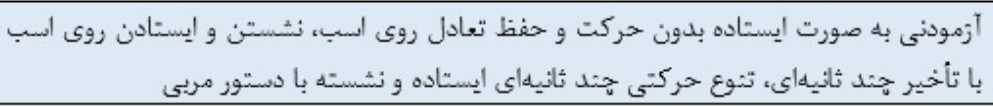 & 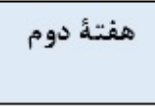 \\
\hline 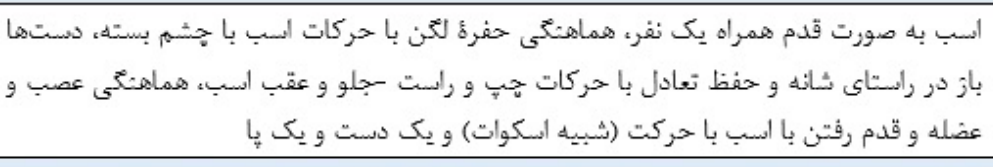 & هفتلة سوم \\
\hline 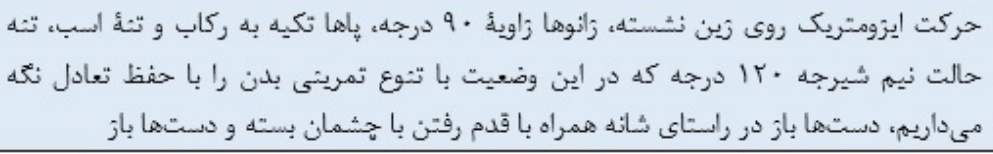 & هفتهُ جحهارم \\
\hline 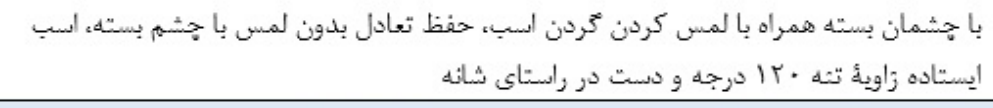 & هفتبة ينجمي \\
\hline با تكيه بر ركاب ايستاده قدم برند، با تثوع حركت و جتد ثائيه ايستاده و نشسته & هفتلهُ ششمم \\
\hline با تعيين مسير حركت اسبب قدم برَند، با تعيين مسير يورثمه زدن & هفتئة هفتم \\
\hline دستها كثار بدن يورتمه برون، بارعايت هماهمثَى حركت اسب و نشستن و ايستادن & هفتئ هشتمم \\
\hline نوائش كيل داخل وخارج -زوازش شانه داخل وخارج -مجرخش كمر و كتف & تمرم كردن \\
\hline حركات كثشى & 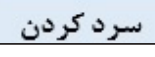 \\
\hline
\end{tabular}

دو ســـح توصيفــى و اســتنباطى و از آزمونهــــاى آمـــارى

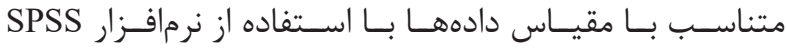

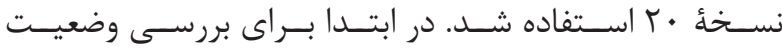

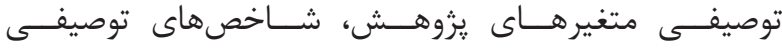

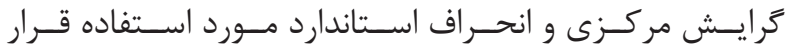

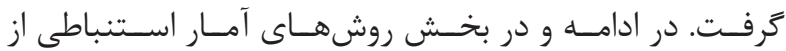

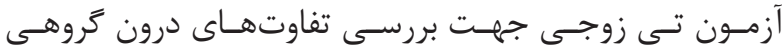

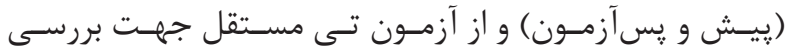

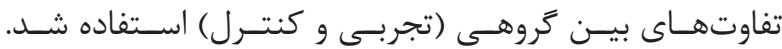

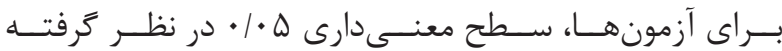

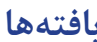

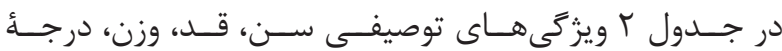

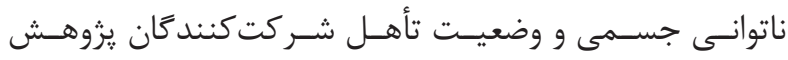

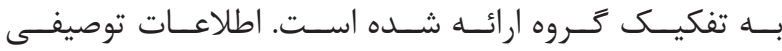

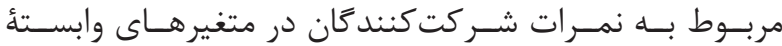

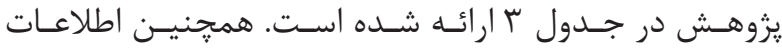

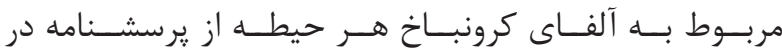

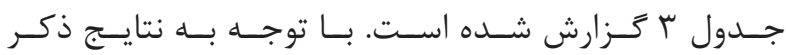

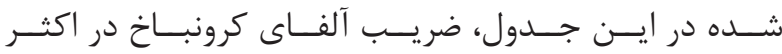

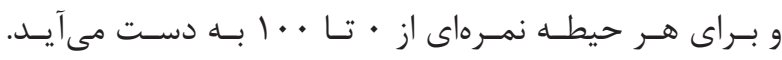

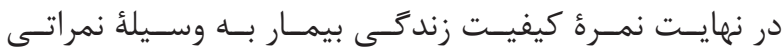

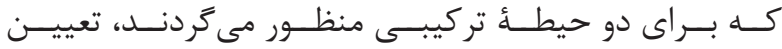

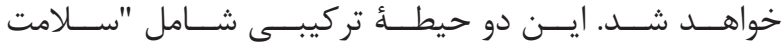

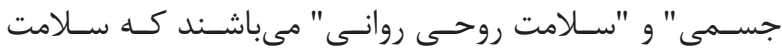

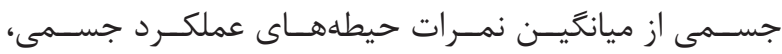

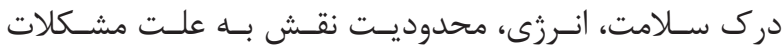

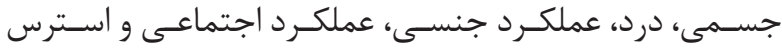

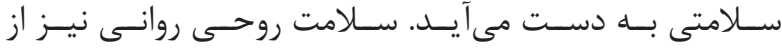

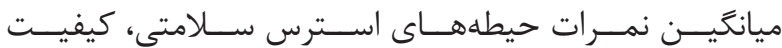

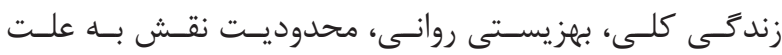

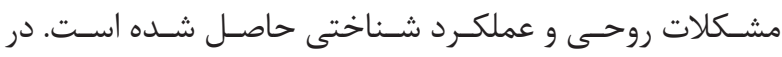

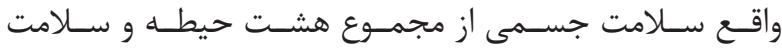

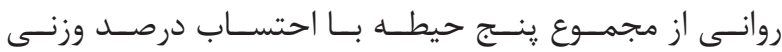

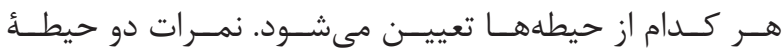

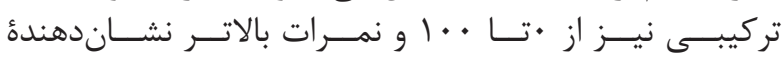

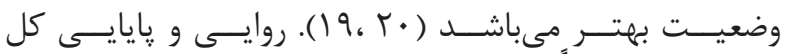

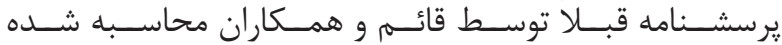

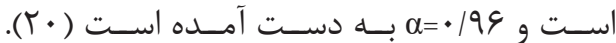
بهمنظــور تجزيــهـ و تحليـل دادههـــاز روشهــــ آمـارى در

جدول r- ميانكين و انحراف معيار شاخصهاى توصيفى شركت كندمًان.

\begin{tabular}{|c|c|c|c|c|c|c|}
\hline وضعيت تأهل & درجهُ ناتواتى & وزن (كيلوكرم) & قد (سانتىمتر ) & سن (سال) & تعداد & كروه \\
\hline ل & $F / r \pm 1 / 4$ & ST/ATI $\pm / T r$ & $|\varepsilon| /|V \pm r / r|$ & $r r / \Lambda r \pm \psi / \cdot \varphi$ & it & تجربى \\
\hline צ ع مجردل & $r / \cdot r \pm r / \Lambda$ & $8 F / 9 \vee \pm 9 / 48$ & $|\& r / \& V \pm F /| F$ & rrET/AG & it & كنترل \\
\hline Iا Iا مجرد & $r / \| \pm r / 1$ & $\varepsilon r / 9 \cdot \pm 9 / \pi)$ & 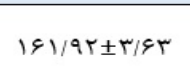 & $r T / E T \pm T / F \Delta$ & re & جمع كل \\
\hline
\end{tabular}




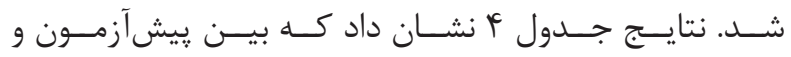

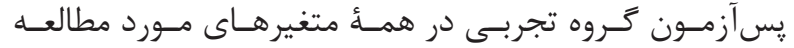

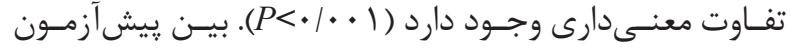

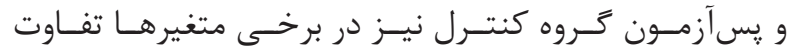

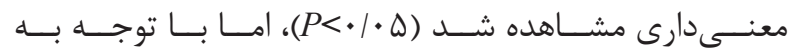

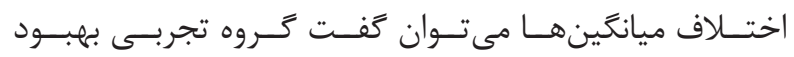

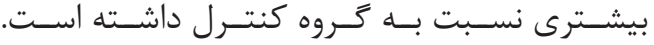

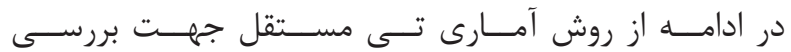

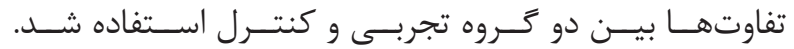

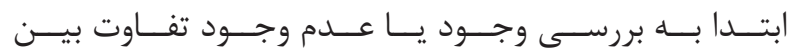

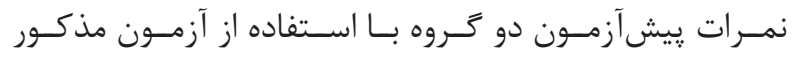

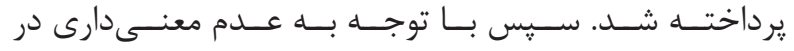

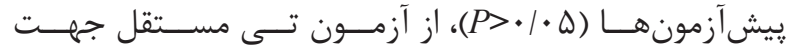

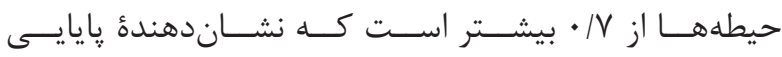

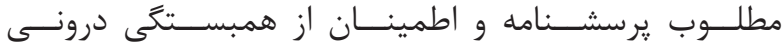

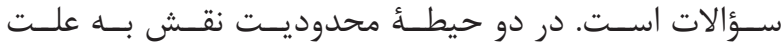

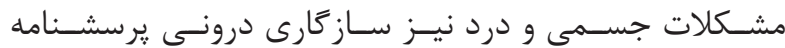

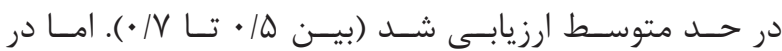

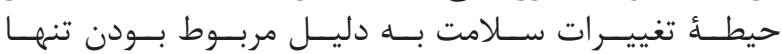

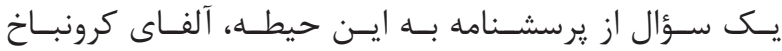

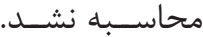

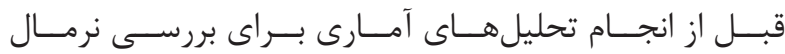

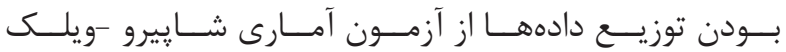

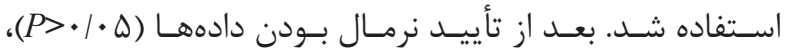

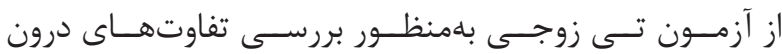

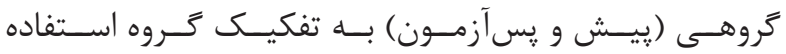

جدول بـ - ميانكَين و انحراف معيار نمرات هر دو كَروه مطالعه در مراحل يِيشآزمون و پِّآزمون و مقادير آلفاى كرونباخ در هر حيطه.

\begin{tabular}{|c|c|c|c|c|}
\hline بسآزمون (MASD) & بيش آزمون (M士SD) & تئو & ضريب آلفاى كرونباخ & متغير هاى وابسته \\
\hline$\Delta \cdot / f T \pm T / / F V$ & $|\Delta / f T \pm| Y / \Delta \mid$ & تجريى & $\cdot / V \Delta$ & \multirow{2}{*}{ حيطل عملكرد جسمى } \\
\hline$r g / T \Delta \pm 9 / \cdot 1$ & $|9 / K \Delta \pm| \cdot / \cdot T$ & كثترل & - & \\
\hline$\Delta T / \cdot \Lambda \pm|\Delta / \Delta|$ & $\mid f / \Delta \Lambda \pm V / A V$ & تجريى & .199 & \multirow{2}{*}{ حيطؤ محدوديت نقش به علت مشكلات جسمى } \\
\hline TT/QTINAN & INVQDVV/OF & كتترل & - & \\
\hline$\Lambda \cdot / \Delta \Delta \pm \mid q / f r$ & $r f / q q \pm 1 \cdot / V T$ & تجريى & $\cdot / V 1$ & \multirow{2}{*}{ حيطة محدوديت نقش به علت مشكلات روحى } \\
\hline 「NКЯ \pm T/TO & $4 t / 4 T \pm Q / V I$ & كثترل & - & \\
\hline $9 q / \cdot r \pm|f /| \mid$ & $r \cdot / \Lambda r \pm / r / r q$ & تجريى & $.19 \mathrm{~V}$ & \multirow{2}{*}{ حيطة درد } \\
\hline TV/DIIY/AF & $r \cdot / 9 v \pm 1 f / r q$ & كتترل & - & \\
\hline$\Delta Q / 9 T \pm I r / V q$ & $F T \pm I r / V A$ & تجريى & $\cdot / \mathrm{VT}$ & \multirow{2}{*}{ حيطلُ بيزيستى روانى } \\
\hline$f r / 1 V \pm 9 / \Delta r$ & $f 1 / r r \pm 9 / 99$ & كثترل & - & \\
\hline$\Delta \Delta \pm|1 / \mathrm{V}|$ & HNGY $\pm 11 / \Delta Y$ & تجريى & $\cdot / V T$ & \multirow{2}{*}{ 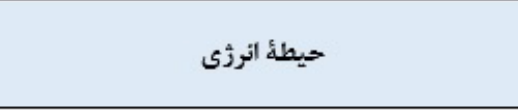 } \\
\hline$f \cdot / T r \pm 1 / / \Delta \varphi$ & $r V \pm 1 / / A r$ & كترل & - & \\
\hline$\Delta N V \Delta \pm 19 / \% q$ & $r q / 9 r \pm l f / f r$ & تجريى & $\cdot / \sqrt{ } 9$ & \multirow{2}{*}{ حيطؤ درك سلامت } \\
\hline 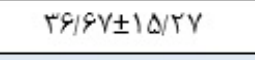 & $r f / \Delta A \pm 1 \Delta / \Delta 9$ & كثترل & - & \\
\hline$g f / \Delta \Lambda \pm r \cdot / f \Delta$ & $r q / f f \pm|r| \Lambda \mid$ & تجريى & $\cdot / \mathrm{VI}$ & \multirow{2}{*}{ حيطك عملكرد اجتماعى } \\
\hline$f r / \wedge q \pm \mid r / \cdot \Delta$ & $f 1 / 11 \pm 1 r / V q$ & كثترل & - & \\
\hline $9 \Delta \pm r \Delta / \Delta \Lambda$ & $F r / 9 r \pm T \cdot / 0$ & تجريى & $\cdot / V Y$ & \multirow{2}{*}{ حيطة تنش سلامت } \\
\hline$f \mid / r \Delta \pm r \cdot / 9$ & KNVDEYT/9G & كثرل & - & \\
\hline$V / r \Delta \pm r / 9 V$ & $F F / Q \Lambda \pm I N G F$ & تجربى & $\cdot / V \Delta$ & \multirow{2}{*}{ حيطة عملكرد شناختى } \\
\hline$f H / T H \pm q / A F$ & $f \mid / 9 V \pm I \cdot / V T$ & ك & - & \\
\hline$\Delta / r \Delta \pm 1 \Delta / \Delta f$ & $r q / \Delta \Lambda \pm 1 \cdot / \lambda V$ & تبحيثى & - & \multirow{2}{*}{ خيطةُ تغيير ات سلامت } \\
\hline FI/GY士IT/KA & $r \Delta / f r \pm I / / \Lambda V$ & كثرل & - & \\
\hline$V G / 9 G \pm \mid f / 10$ & $f \Delta \pm I f / V T$ & تجمبيى & $\cdot / V T$ & \multirow{2}{*}{ حيطة كيفيت زندثى كل } \\
\hline$f f / 99 \pm 9 / / 4 f$ & $f r / \cdot \Lambda \pm 1 \cdot \mid G Y$ & ك & & \\
\hline$\Delta r / q \pm|r / r|$ & YNGFENFT & تجمرئى & $\cdot / \lambda f$ & \multirow{2}{*}{ حيطة تركيبى سلامت جسمى كيفيت زندثى } \\
\hline$r T / Y) \pm f(Q)$ & $r V / 9 r \pm f / Q T$ & كثرل & & \\
\hline$V \cdot / r V \pm I f / V f$ & $r q / T V \pm I r / f r$ & تجربى & $\cdot / 19$ & \multirow{2}{*}{ حيطة تركيبى سلامت روحى -روانى كيفيت زندكى } \\
\hline Fr/TIDS/fG & $r G / A V \pm V / f r$ & ك-5 & & \\
\hline
\end{tabular}


بحث و نتيجه

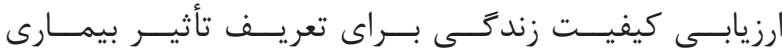

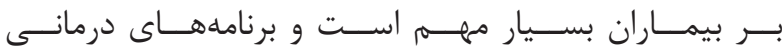

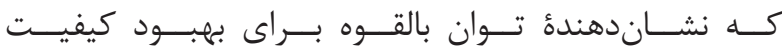

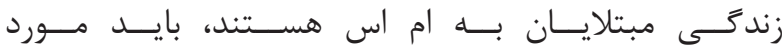

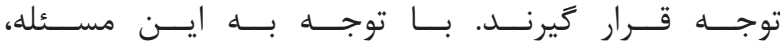

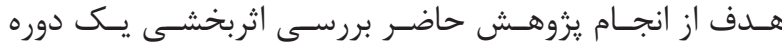

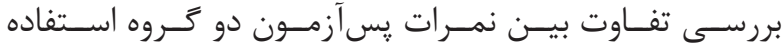

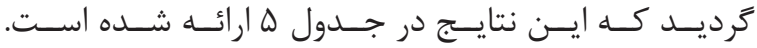

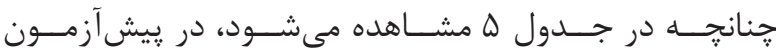

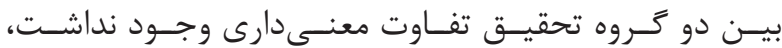

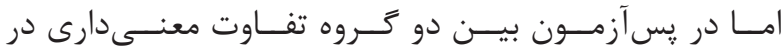

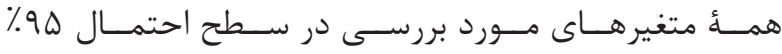

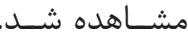

جدول \&- خلاصه نتايج آزمون تى زوجى براي مقايسٔ نمرات بيش و يسآزمون به تفكيك كروه.

\begin{tabular}{|c|c|c|c|c|c|c|}
\hline معنى سطارى & 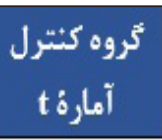 & تفائكين & معنى سطارى & آمروه تجربى & ميأثَين & مثثير هاى وابسته \\
\hline$\cdot \cdots 1$ & $9 / \pi \Lambda$ & 1. & $\cdot \mid \cdot 1$ & NIT & ro & حيطةُ عملكرد جسمى \\
\hline .1199 & $1 / f \wedge$ & $f / I V$ & $\cdot \mid \cdot 1$ & $9 / 01$ & $r v / Q$. & حيطة محدوديت ثقش به علت مشكلات جسمى \\
\hline$\cdot \cdot \cdot \gamma$ & r/Tr & $1919 \mathrm{~V}$ & $\cdot \mid \cdot \cdot$ & $\Delta / \wedge 9$ & $\Delta \Delta / \Delta \varphi$ & حيطةُ محدوديث نقش به علت مشكلات روحى \\
\hline$\cdot \cdots 1$ & $\Delta / \Delta \Lambda$ & $9 / 0 \mathrm{~T}$ & $\cdot \cdot \cdots 1$ & $1 \% / f \Delta$ & rN/19 & 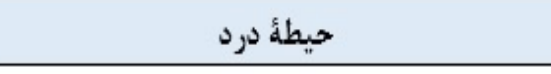 \\
\hline$\cdot \cdot \cdot 1$ & $V / 9)$ & l/Ar & $\cdot \cdot \cdot 1$ & $\Delta / A r$ & $19 / 91$ & حيطة بهزيستى رواثى \\
\hline$\cdot 1 \cdot .1$ & $11 / \mathrm{rr}$ & r/rr & $\cdot \mid \cdot \cdot$ & $\Delta / f r$ & $19 / 4$ & حيطةُ انرزى \\
\hline $.1 \cdot 1 \mathrm{\gamma}$ & $r / \Lambda$. & $r / \cdot \Lambda$ & $\cdot \cdot \cdot \cdot 1$ & V/9. & INAT & حيطةُ درك سلامث \\
\hline $.1 \cdot 1 Y$ & $r / \Lambda$. & $r / V \Lambda$ & $\cdot \cdots 1$ & $\Delta / 91$ & to/lf & حيطةُ عملكرد اجتماعى \\
\hline$\cdot \cdot \cdot \gamma$ & r/Tr & $r / 0 \cdot$ & $\cdot \mid \cdot 1$ & $\Delta / 1$. & $r t / \cdot 1$ & حيطة تنش سلامت \\
\hline$\cdot 1 \cdot 49$ & $t / r f$ & $1 / 9 \mathrm{~V}$ & $\cdot \cdot \cdots 1$ & NAf & $r 9 / 9 \mathrm{~V}$ & 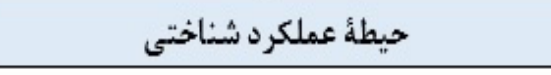 \\
\hline$\cdot \cdot \cdot \lambda r$ & 1/91 & $9 / \pi \Delta$ & $\cdot \mid \cdot \cdot 1$ & V/fr & $f 1 / 9 \mathrm{~V}$ & حيطةُ تغييرات سلامت \\
\hline$\cdot 1 \cdot 41$ & $r / 99$ & $r / M$ & $\cdot 1 \cdots 1$ & $1 \cdot / V \Lambda$ & r1/99 & حيطؤ كيفيث زندكى كل \\
\hline$\cdot \cdots 1$ & $9 / 49$ & $f / T \Lambda$ & $\cdot \cdot \cdot 1$ & $1 \cdot \pi r$ & ro/rG & حيطؤ تركيبى سلامت جسمى كيفيت زندكى \\
\hline$\cdot \cdot \cdot 1$ & $\Delta(r)$ & $\Delta / 90$ & $\cdot 1 \cdot \cdot 1$ & $9 / \pi 9$ & $r 1 / \cdot r$ & حيطةُ تركيبى سلامت روانى كيفيت زندكى \\
\hline
\end{tabular}

جدول ه- خلاصه نتايج آزمون تى مستقل براى مقايسة نمرات بِيش و پسآزمون بين دو گروه مستقل.

\begin{tabular}{|c|c|c|c|c|}
\hline \multicolumn{2}{|c|}{ بسآزمون } & \multicolumn{2}{|c|}{ بيش آزمون } & \multirow{2}{*}{ متغيرهاى وايسته } \\
\hline سطح معنى دارى & t آمارة & سطج معنى دارى & t آمارة & \\
\hline$* H$ & $r / \Delta 9$ & $\cdot / 1 \Delta 9$ & $\cdot / 11$ & حيطة عملكرد جسمى \\
\hline$\cdot 1+\cdots 1$ & r/q. & - fat & $\cdot / \mathrm{VI}$ & حيطة محدوديت نقش به علت مشكلات جسمى \\
\hline$\cdot+\cdots)$ & $F / F)$ & - IVor & $\cdot / Y T$ & حيطه محدوديت نقش به علت مشكلات روحى \\
\hline$\cdot(*)$ & $\Delta / V Y$ & $\cdot / 911$ & $\cdot l \cdot t$ & حيطة درد \\
\hline$\cdot 1+\cdots$ & r/SF &.$/ R T$ &.$/ 48$ & حيطةٌ بهزيستى روانى \\
\hline$\cdot 1+\theta$ & $r / 9$ & $\cdot|V F|$ & $\cdot / Y T$ & حيطة انرزى \\
\hline 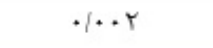 & $r / F)$ & $\cdot / Y 9 F$ & $\cdot[A V$ & حيطة درك سلامت \\
\hline$\cdot 1 \cdots 1$ & $r / 9 \Delta$ & $\cdot / \mathrm{rr} \cdot$ & $\cdot / r$. & حيطهُ عملكرد اجتماعى \\
\hline$+(+4)$ & r/Fq & .1948 & $\cdot / \% \Lambda$ & حيطةٌ تنش سلامت \\
\hline $.1+r$ & r/AV &.$/ S F Y$ & $\cdot / F V$ & حيطهُ عملكرد شناختى \\
\hline$\cdot 1 \cdots 1$ & $9] \cdot 9$ &.$/ 4 Y 4$ & $\cdot / \mathrm{Vq}$ & حيطة تغييرات سلامت \\
\hline$\cdot 1 \cdots)$ & S/FV & $\cdot / \Delta \wedge r$ & $\cdot 109$ & حيطة كيفيت زندكى كل \\
\hline$\cdot \mid+\cdots$ & $\Delta / r_{\lambda}$ & $\cdot / \Lambda \cdot \cdot$ &.$/ 48$ & حيطة تركيبى سلامت جسمى كيفيت زندوىى \\
\hline$\cdot 1+\cdots$ & $9] \cdot F$ & - $\operatorname{cor} \Delta$ &.$/ 9 F$ & حيطة تركيبى سلامت روانى كيفيت زندّى \\
\hline
\end{tabular}




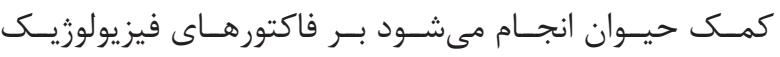

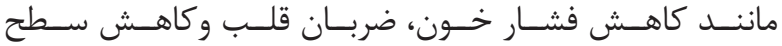

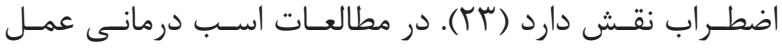

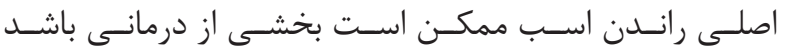

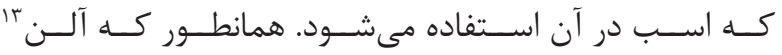

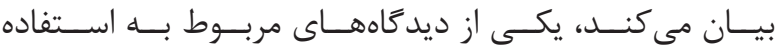

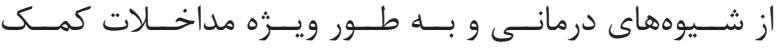

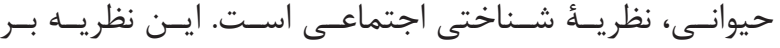

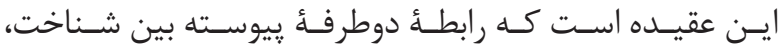

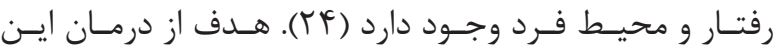

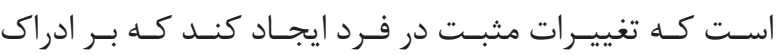

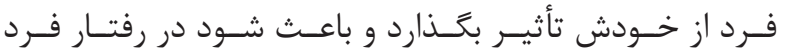

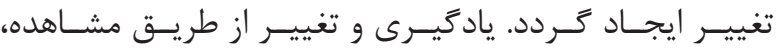

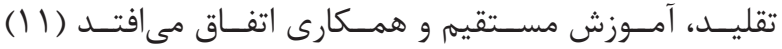

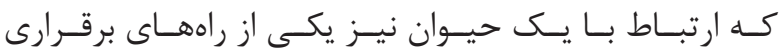

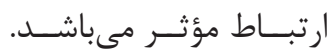

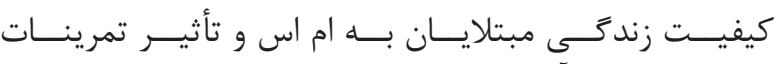

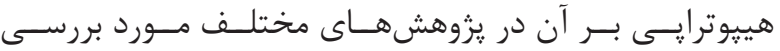

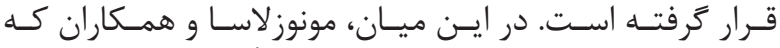

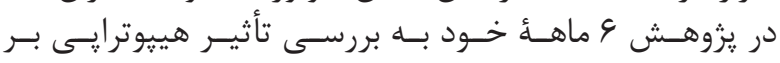

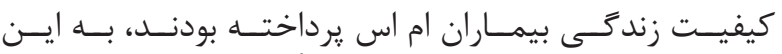

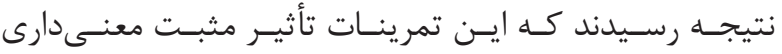

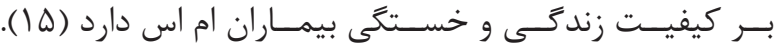

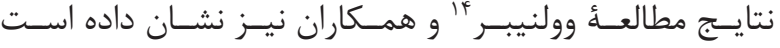

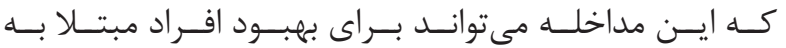

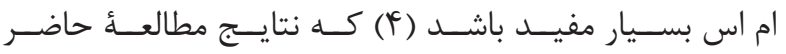

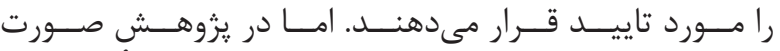

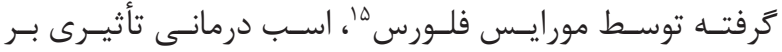

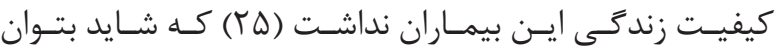

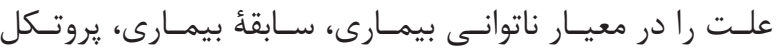

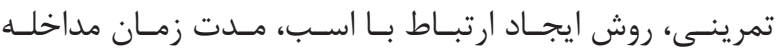

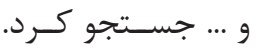

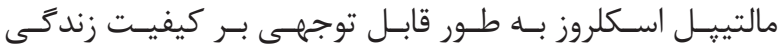

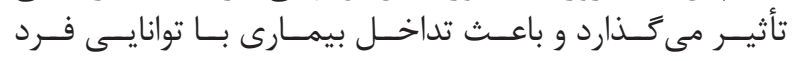

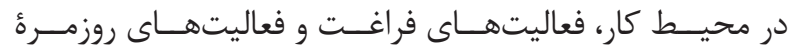

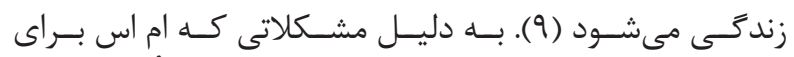

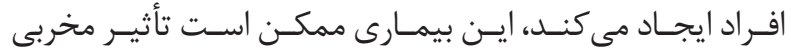

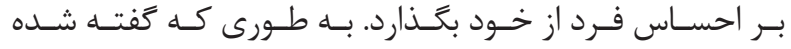

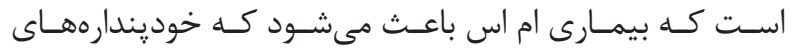

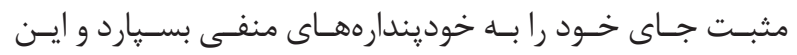

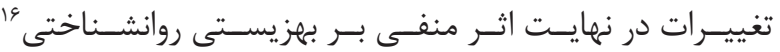

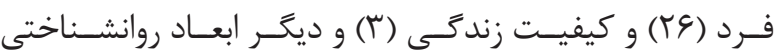

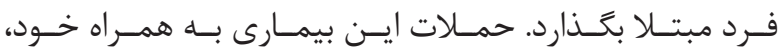

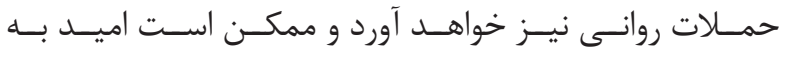

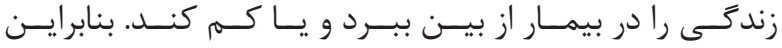

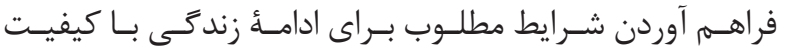

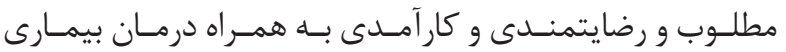

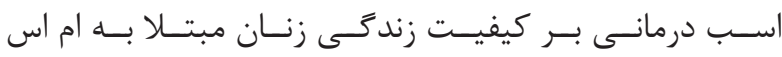

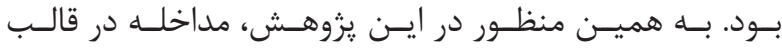

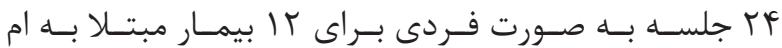

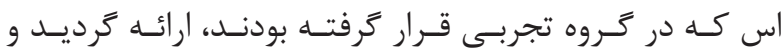

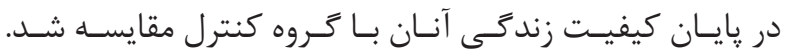

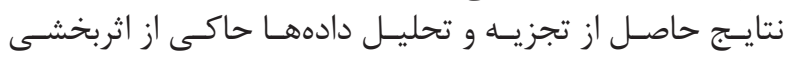

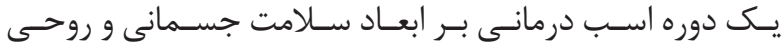

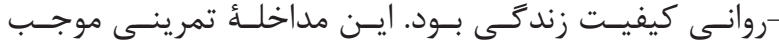

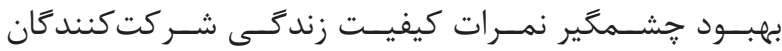

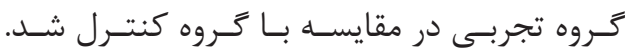

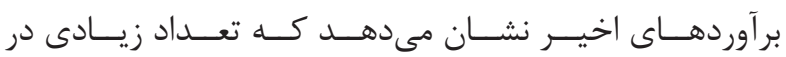

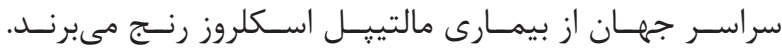

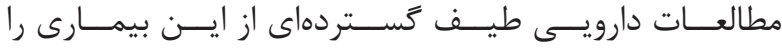

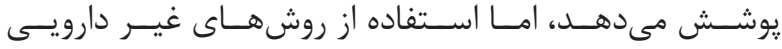

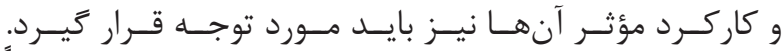

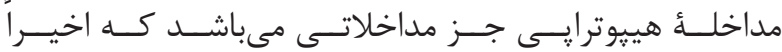

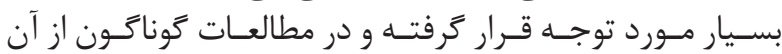

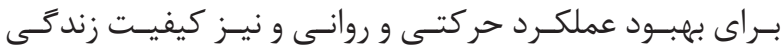

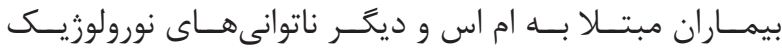

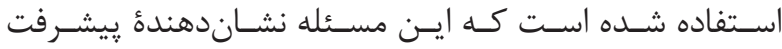

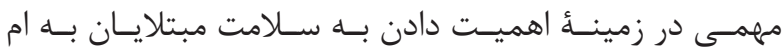

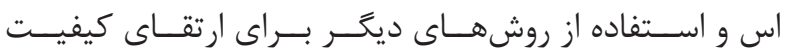

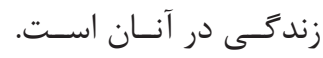

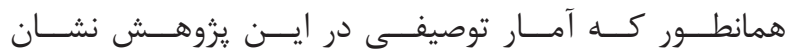

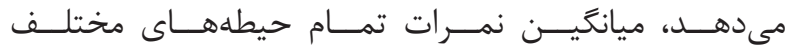

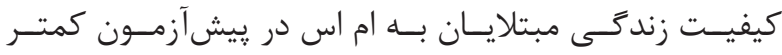

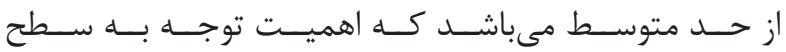

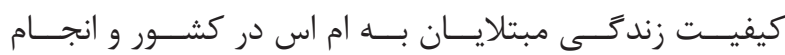

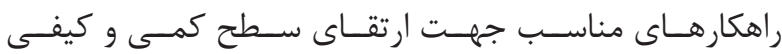

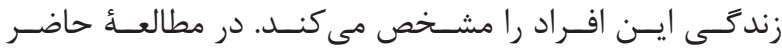

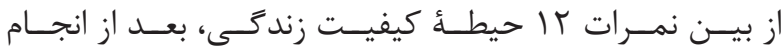

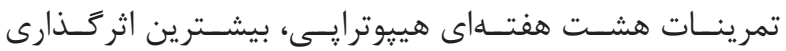

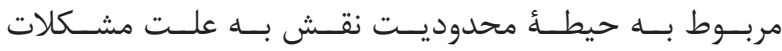

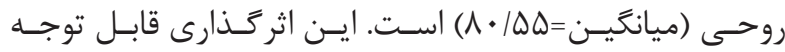

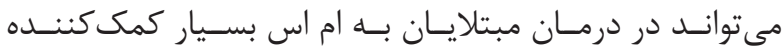

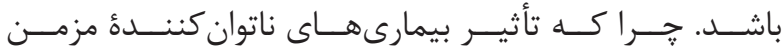

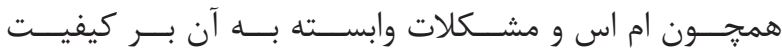

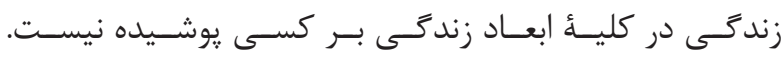

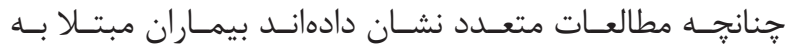

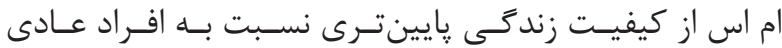

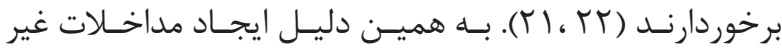

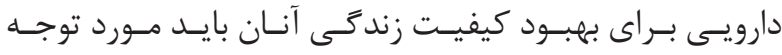

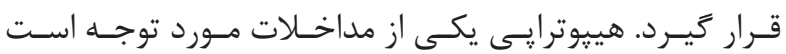

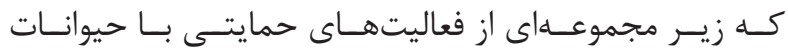

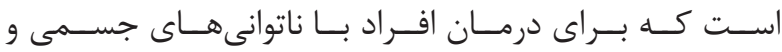

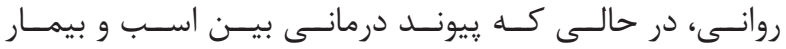

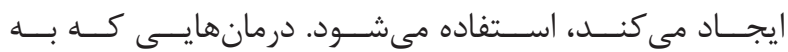




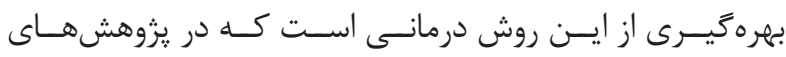

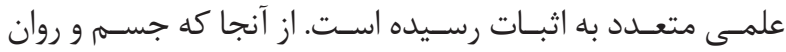

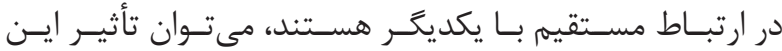

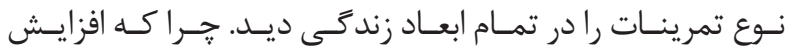

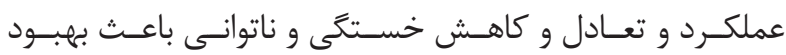

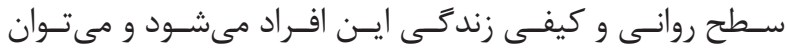

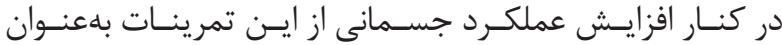

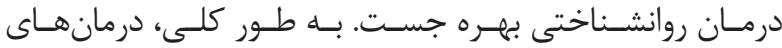

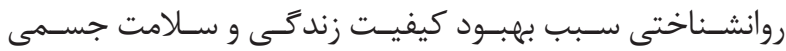

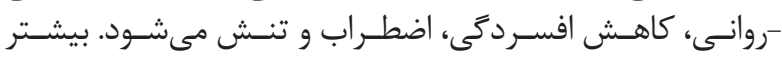

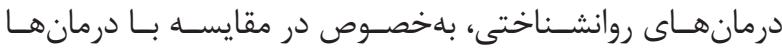

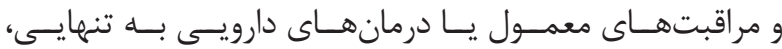

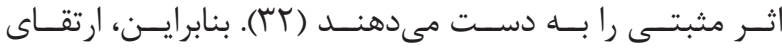

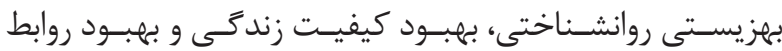

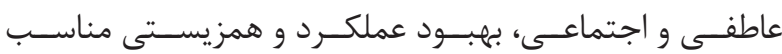

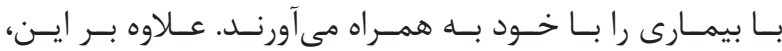

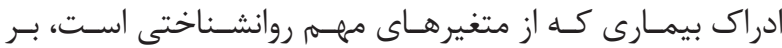

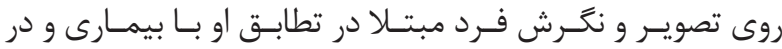

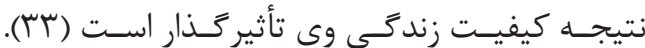

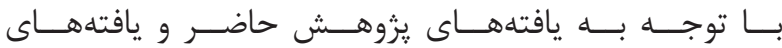

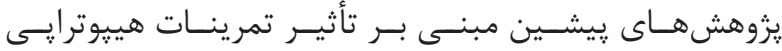

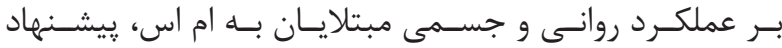

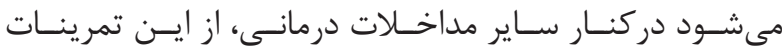

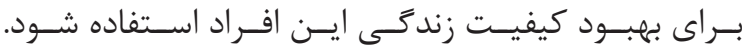

1. Dusti F, Frughipur M, Sohrabi M, Taheri HR, Saeidi M, Tymuri S. Effect of Instructions for Internal and Far and Near External Focus of Attention on Balance of Multiple Sclerosis Patients. Medical Journal of Mashhad University of Medical Sciences. 2013; 56(1): 15-20.

2. Cameron MH, Nilsagard YE. Measurement and treatment of imbalance and fall risk in multiple sclerosis using the international classification of functioning, disability and health model. Phys Med Rehabil Clin N Am. 2013; 24(2): 337-54.

3. Mami Sh, Heidary M. Effectiveness of intensive short-term dynamic psychotherapy on sexual function and emotional expression in women with multiple sclerosis. Quarterly Journal of Health Psychology. 2019; 7(28): 131-50.

4. Wollenweber V, Drache M, Schickendantz S, Gerber Grotea A, Schiller P, Pohlau D. Study of the effectiveness of hippotherapy on the symptoms of multiple sclerosis - Outline of a randomised controlled multicentre study (MS-HIPPO). Contemp Clin Trials Commun. 2016; 3: 6-11.

${ }^{16}$ Psychological well-being

${ }^{17}$ So

${ }^{18}$ Hong and Yoon

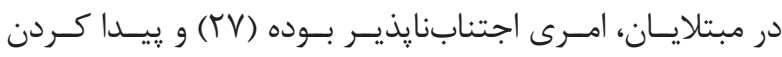

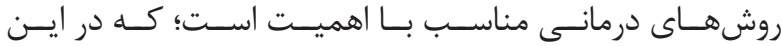

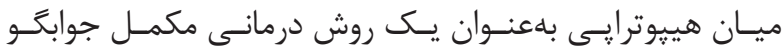

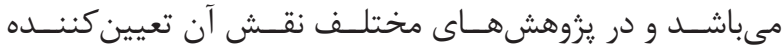

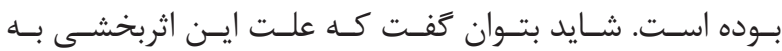

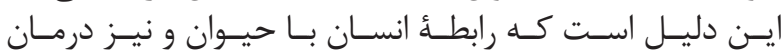

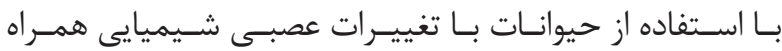

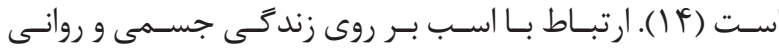

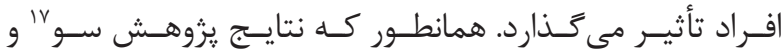

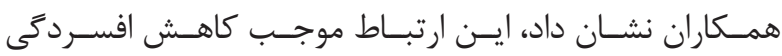

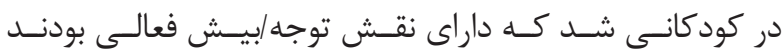

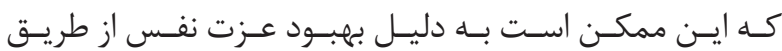

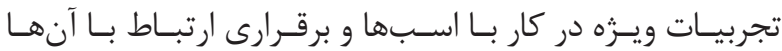

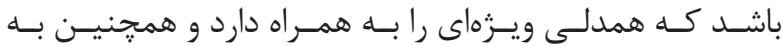

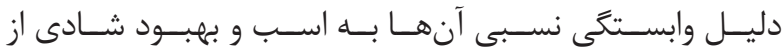

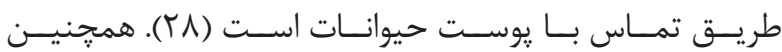

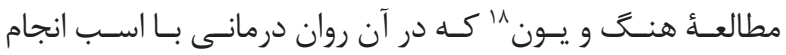

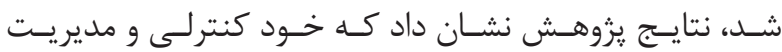

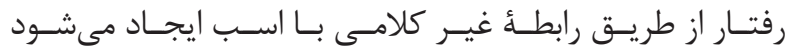

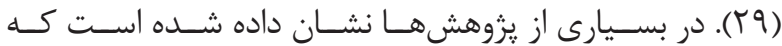

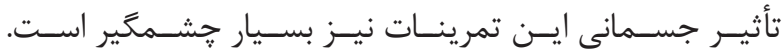

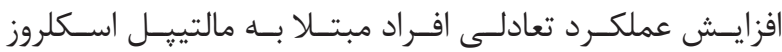

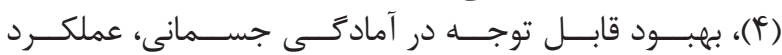

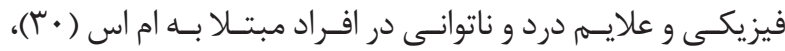

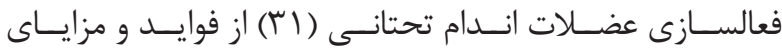

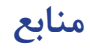

5. Javanmard GH. The comparison of performance of prospective/ retrospective, short-term/ long-term, and allocentric/ egocentric memory in ms patients and healthy individuals. Quarterly Journal of Health Psychology. 2017; 6(22): 5-19.

6. Manafi SF, Dehshiri Gh. Fear of disease progression in patients with cancer and multiple sclerosis and its relation to emotional problems. Quarterly Journal of Health Psychology. 2017; 6(22): 115-30.

7. Chiaravalloti ND, DeLuca J. Cognitive impairment in multiple sclerosis. Lancet Neurol. 2008; 7(12): 1139-51.

8. Sangelaji B, Salimi Y, Dastoorpour M, Mansouri T, Ashrafinia F, Esmaeilzadeh N, et al. The relationship between disability and quality of life in multiple sclerosis patients. Journal of Health \& Development. 2013; 2(3): $203-13$.

9. Ysrraelit MC, Fiol MP, Gaitan MI, Correale J. Quality of life assessment in multiple sclerosis: different perception between patients and neurologists. Front Neurol. 2018; 8: 729. doi: 10.3389/fneur.2017.00729. 


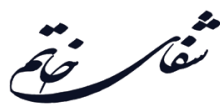

10. Pauw J. Therapeutic horseback riding studies: problems experienced by researchers. Physiotherapy. 2000; 86(10): 523-27.

11. Saremianfar M, Movahedi AR, Rafei Brojeni M, Najafi M. The effect of instructional horseback riding on social interaction of children with Autism spectrum disorders. Journal of Motor Behavior. 2015; 7(21): 33-46.

12. Dietert RR, Dietert JM, Dewitt JC. Environmental risk factors for autism. Emerg Health Threats J. 2011; 4.

13. Evans N, Gray C. The practice and ethics of animalassisted therapy with children and young people: is it enough that we don't eat our co-workers? British J Social Work. 2012; 42(4): 600-17.

14. Munoz Lasa S, Maximo Bocanegra N, Valero Alcaide R, Atin Arratibel MA, Varela Donoso E, Ferriero G. Animal assisted interventions in neurorehabilitation: a review of the most recent literature. Neurologia. 2015; 30(1): 1-7.

15. Munoz Lasa S, Lopez de Silanes C, Atin Arratibel MA, Bravo Llatas C, Pastor Jimeno S, Maximo Bocanegra N. Effects of hippotherapy in multiple sclerosis: pilot study on quality of life, spasticity, gait, pelvic floor, depression and fatigue. Med Clin (Barc). 2019: 152(2): 55-8.

16. Seredova M, Maskova A, Mrstinova M, Volicer L. Effects of hippotherapy on well-being of patients with schizophrenia. Archives of Neuroscience. 2016: 3(4): e39213.

17. Giglio CP. Accessibility of animal-assisted therapy for children with autism: a parent's perspective: a project based upon an independent investigation. Theses, Dissertations, and Projects. 2010.

18. Babicz A, Donoho H, Hoven M, Jones A, Moravetz M, Morrow M, et al. The effectiveness of hippotherapy on perceived quality of life for preschool aged children with down syndrome through parent report. Utica College, ProQuest Dissertations Publishing. 2018.

19. Choobforouszade A, Neshatdoos HT, Molavi H, Abedi MR. Examine the quality of life among Multiple sclerosis patients in Yazd MS association,Iran. The Journal of Toloo-e-behdasht. 2016; 14(5): 131-40.

20. Ghaem H, Borhani Haghighi A, Jafari P, Nikseresht AR. Validity and reliability of the Persian version of the multiple sclerosis quality of life questionnaire. Neurol India. 2007; 55(4): 369-75.

21. Tepavcevic DK, Kostic J, Basuroski ID, Stojsavjevic N, Pekmezovic T, Drulovic J. The impact of sexual dysfunction on the quality of life measured by MSQoL-54 in patients with multiple sclerosis. Mult Scler. 2008; 14(8): 1131-6.

22. Messmer Uccelli M, Traversa S, Ponzio M. A survey study comparing young adults with MS and healthy controls on self-esteem, self-efficacy, mood and quality of life. J Neurol Sci. 2016; 368: 369-73.

23. Morrison ML. Health benefits of animal-assisted interventions. Journal of Evidence-Based Integrative Medicine. 2007; 12(1): 51-62.

24. Allen BP. Personality theories: development, growth, and diversity. 3rd ed. Boston, MA: Allyn and Bacon. 2000; p. 238-50.

25. Moraes Flores F, Santos Sousa L, Mendona Menezes K, Copetti F, Morais Trevisan C. Quality of life in multiple sclerosis patients participating in therapeutic horseback riding. ConScientiae Saude. 2014; 13(1): 39-46.

26. Barker A, das Nair R, Lincoln N, Hunt N. Social identity in people with multiple sclerosis: a metasynthesis of qualitative research. Social Care and Neurodisability. 2014; 5(4): 256-67.

27. Famil Sharifian Y, Khalili M, Hasanzadeh Pashang S, Kamarzarin H, Taghizadeh ME. The study of efecttiveness of cognitive-behavioral therapy in group on mental health among primary MS patients. Journal of Urmia Medical. 2013; 24(9): 652-64.

28. So WY, Lee SY, Park Y, Seo DI. Effects of 4 weeks of horseback riding on anxiety, depression, and self-esteem in children with attention deficit hyperactivity disorder. Journal of Men's Health. 2017; 13(2): 1-7.

29. Hong JY, Yoon JH. Study for equine facilitated psychotherapy. J Play Ther. 2009; 13: 99-111.

30. Edwards T, Pilutti LA. The effect of exercise training in adults with multiple sclerosis with severe mobility disability: a systematic review and future research directions. Mult Scler Relat Disord. 2017; 16: 31-9.

31. Ribeiro MF, Espindula AP, Bevilacqua Junior DE, Tolentino JA, Silva CFRD, Araujo MF, et al. Activation of lower limb muscles with different types of mount in hippotherapy. J Bodyw Mov Ther. 2018; 22(1): 52-6.

32. Pagnini F, Bosma CM, Phillips D, Langer E. Symptom changes in multiple sclerosis following psychological interventions: a systematic review. BMC Neurol. 2014; 14: 222. doi: 10.1186/s12883-014-0222-z.

33. Shamili F, Zare H, Oraki M. The predicting quality of life based on illness perception in multiple sclerosis patients. The Journal of Urmia University of Medical Sciences. 2013; 24(6): 379-92. 